\title{
Modelos geo-espaciales para la vigilancia local de la salud
}

\author{
Diana Elba De Pietri, ${ }^{1}$ Susana García ${ }^{2}$ y Osvaldo Rico ${ }^{3}$
}

Forma de citar De Pietri DE, García S, Rico O. Modelos geo-espaciales para la vigilancia local de la salud. Rev Panam Salud Publica. 2008;23(6):394-402.

RESUMEN Objetivos. Generar un modelo geo-espacial para evaluar la exposición a plomo de escolares de 6 a 8 años, residentes de San Antonio Oeste, en la provincia de Río Negro, Argentina, localidad contaminada por una fundición en el casco de la ciudad y la acumulación a cielo abierto de sus residuos.

Métodos. El análisis espacial llevado a cabo de octubre a abril de 2006 incluyó la interpretación satelital y el georreferenciamiento de los datos para la espacialización de la información. Se tomó en cuenta la localización de las residencias sobre calles de tierra y la proximidad, desde el domicilio y/o la escuela de los niños bajo estudio, a los sitios-fuente como puntos de exposición. Resultados. Las muestras de sangre tomadas a los niños en las escuelas en el área de influencia de alguna fuente, mostraron mayor abundancia de plombemias elevadas que las localizadas en otras áreas. Dichas plombemias se asociaron con la cercanía a alguna fuente y/o la ubicación de las residencias sobre calles de tierra. Los valores más altos de plomo en sangre correspondieron a mayores rangos de plomo ambiental.

Conclusiones. El análisis espacial constituyó una metodología complementaria útil para el análisis y la gestión de riesgo del sitio contaminado, al indicar una asociación positiva entre plombemias elevadas y la proximidad a la fuente, y/o el hecho de vivir en calles de tierra, que no se había hecho evidente mediante los análisis epidemiológicos tradicionales. Los resultados aportaron la evidencia científica necesaria para indicar la implementación de medidas de intervención directas sobre las fuentes de exposición y de educación, orientadas a fomentar hábitos dietéticos higiénicos en la población.

Palabras clave Sistemas de Información Geográfica, exposición a riesgos ambientales, sangre, plomo, salud infantil, Argentina.

La aplicación de un sistema de información geográfica (SIG) como técnica para analizar la situación de salud

\footnotetext{
1 Ministerio de Salud de Nación-Epidemiología. La correspondencia se debe dirigir a Diana Elba de Pietri, Ministerio de Salud de Nación-Epidemiología. Av. 9 de julio 1925, Buenos Aires C1073ABA, Argentina. T.: 5411 43799237. F.: 541143799127. Correo electrónico: dpietri@msal.gov.ar

2 Ministerio de Salud de Nación-Promoción de la salud. Buenos Aires, Argentina.

3 Ministerio de Salud de Nación. Buenos Aires, Argentina.
}

y las condiciones de vida de una localidad determinada, presenta poca sistematización en el proceso de gestión de riesgo. No obstante, con la utilización de estrategias de información y comunicación apropiadas, este tipo de sistemas pueden resultar de gran utilidad, particularmente en regiones vulnerables (1-4).

En este trabajo se aborda la aplicación del SIG a la problemática de salud ambiental de la localidad de San Anto- nio Oeste (SAO), en la provincia de Río Negro, Argentina, donde una serie de estudios demostraron la existencia de contaminación en suelo, polvo doméstico, sedimentos y agua, con arsénico, plomo, zinc, cobre, hierro y cadmio, entre otros elementos (5-8). El problema se debe tanto al acopio de materiales de la mina Gonzalito, a $120 \mathrm{~km}$ de distancia de SAO, y que posteriormente son enviados a Buenos Aires, como a las actividades de fundi- 
ción que se realizan desde 1965 dentro del casco urbano, con la acumulación de sus residuos a cielo abierto a $2 \mathrm{~km}$ de distancia de la ciudad.

En consideración a su impacto deletéreo sobre la salud infantil y debido a la existencia de técnicas que reducirían los efectos de la contaminación, la exposición ambiental a plomo ocupa un lugar prioritario en materia de salud pública. La determinación de plomo en sangre o plombemia, es el método idóneo para evaluar la exposición a plomo y definir las medidas de intervención ambiental y terapéuticas que habrán de implementarse. La plombemia constituye un biomarcador de exposición que refleja, asimismo, la interacción entre la absorción del metal en el organismo, su distribución en tejidos blandos, el depósito en tejido óseo y la filtración renal.

Las plombemias iguales o mayores a $10 \mu \mathrm{g} / \mathrm{dL}$ se consideran como elevadas y están potencialmente asociadas a déficit neurológico infantil (9). Al no existir valores de referencia para población infantil expuesta que sean aceptados por el Ministerio de Salud de la Nación, se han adoptado los estándares del Centro de Control de Enfermedades de los Estados Unidos de América. Cabe destacar que en esta última nación hace ya varias décadas que se iniciaron los programas para la erradicación de la contaminación con plomo $(10,11)$ y que actualmente dichos valores son cuestionados por expertos que sostienen que no pueden considerarse como normales las plombemias superiores a $5 \mu \mathrm{g} / \mathrm{dL}(12,13)$ debido a que, si bien hay discrepancias, el cociente intelectual puede disminuir de 0,25 a 0,5 puntos por cada microgramo de plomo que aumenta en la plombemia.

En este contexto, el Ministerio de Salud de la Provincia de Río Negro, junto con el municipio de SAO y el grupo de trabajo formado por organizaciones intermedias de la comunidad, con la colaboración del Ministerio de Salud de la Nación, realizaron entre agosto y noviembre de 2005 un estudio para conocer el riesgo de exposición ambiental a plomo en escolares de dicha localidad. En este trabajo se muestra el análisis espacial desarrollado en el estudio de SAO, cuyo objetivo fue la generación de un modelo geoespacial, a partir de datos de salud, que pudiera utilizarse en la evaluación de los factores de exposición.

\section{MATERIALES Y MÉTODOS}

Para llevar a cabo el análisis espacial fue necesario cumplir previamente con una serie de etapas a cargo de diferentes equipos de trabajo; la secuencia de tareas se describe a continuación.

\section{Reconocimiento en terreno}

En octubre de 2005 se visitó la localidad de SAO para identificar y ubicar las posibles fuentes de contaminación ambiental. La georreferenciación de las fuentes potenciales de plomo ambiental se realizó sobre una imagen ASTER.

\section{Acceso y adecuación de la información}

Cartografía e información censal. Se adquirió el amanzanado digital, así como los ejes de calles de la ciudad, junto con los datos de población y vivienda del último censo nacional (14). Dicha información se utilizó como base para los análisis posteriores. Se emplearon los programas SIG-Epi (15, 16) y ArcView GIS. The Geographic Information System for Everyone. Version 3.0 for Windows. ESRI 1992-1997.

Población estudiada. Los datos que proporcionó la Dirección de Epidemiología del Ministerio de Salud de la Nación corresponden a un muestreo aleatorio, realizado por escuela, en la localidad de SAO. Se utilizó un diseño descriptivo de corte transversal. La muestra se fijó en 200 escolares de 6 a 8 años de edad, pero en el estudio sólo se incluyeron 195 escolares por aceptación voluntaria de los padres. Sin embargo, el análisis espacial se hizo con 186 domicilios de residencia, además de las escuelas a las cuales asisten los niños y los resultados de los análisis de plomo en sangre correspondientes a las muestras de sangre obtenidas en febrero de 2006.

La plombemia se determinó con la técnica de espectrofotometría de absorción atómica en horno de grafito. Se determinó la concentración de hemoglobina y hematocrito. Los niveles de plombemia se clasificaron según el tipo de intervención recomendada, y se definieron como casos de plombemia elevada aquellos con valores iguales o superiores a $10 \mu \mathrm{g} / \mathrm{dL}(17)$.

Plomo ambiental. Se obtuvo información del Centro Nacional Patagónico sobre un estudio ambiental realizado en noviembre de 2005, que consistió en un análisis de 35 muestras de polvo ambiental ubicadas aleatoriamente en la matriz urbana, y que se recolectaron en las veredas "barriendo" con brochas limpias el sector más transitado. El polvo se guardó en bolsas para su traslado al laboratorio. Los análisis de metales se llevaron a cabo por espectrofotometría de absorción atómica.

\section{Georreferenciación}

Los 186 domicilios de los niños estudiados se ubicaron sobre la base cartográfica digital, por manzana, de la localidad de SAO. Asimismo se georreferenciaron las seis escuelas de $\mathrm{SAO}$, la ubicación de las posibles fuentes de contaminación, la localización de sitios de toma de muestras ambientales y las calles según tuvieran o no carpeta asfáltica.

En esta etapa se generaron los siguientes mapas temáticos: 1) mapa de puntos con la localización de los domicilios de los niños a quienes se les extrajo sangre; 2) mapa de puntos con la ubicación de las escuelas de SAO; 3) mapa de polígonos con la localización de las posibles fuentes de contaminación; 4) mapa de puntos ubicando los sitios de toma de muestras ambientales, y 5) mapa de líneas indicando la cobertura de calles de asfalto (figura 1). 
FIGURA 1. Secuencia de mapas elaborados para el análisis espacial, Argentina, 2006

Mapas incicales

\begin{tabular}{|l|}
\hline $\begin{array}{l}1 . \\
\text { Mapa de puntos con } \\
\text { domiclios de } \\
\text { residencia de los } \\
\text { niños a quienes se } \\
\text { les extrajo sangre. }\end{array}$ \\
\begin{tabular}{|l}
2. \\
Mapa de puntos con \\
localización de las \\
escuelas.
\end{tabular} \\
\hline
\end{tabular}

3.

Mapa de polígonos

con fuentes de contaminación

capturados de la

imagen satelital.

4.
Mapa de puntos con
domicilios de
muestras ambien-
tales.

\section{5.}

Mapa de líneas indicando la cobertura de calles de asfalto.
Mapas derivados

Mapas interpretados

1.1 .1

Distribución de plombemias deseadas y

1.2 Mapa de líneas que representan los valores de plombemia similares.

1.3 Mapa continuo de valores estimados de plombemia. no deseadas.

1.3.1

Focos con plombemias no deseadas.

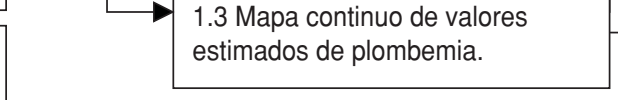

(1)

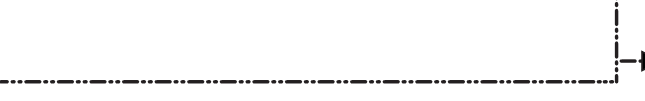

2.1.1 Mapa de puntos clasificados según escuela a la cual asiste cada uno de los niños muestreados.

3.1 Mapa continuo con distancias concéntricas alrededor de las fuentes de contaminación.

3.1.1

Mapa con áreas de influencia de cada fuente hasta 400 metros.

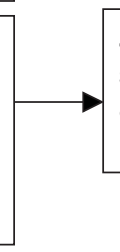

4.1 Mapa de líneas que representan similares valores de plomo en el polvo ambiental.

4.2 Mapa continuo de valores estimados de plomo ambiental.

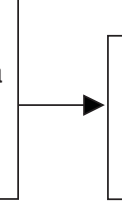

5.1 Mapa continuo con distancias alrededor de las calles de asfalto.
5.1.1

Mapa con áreas de

influencia de calles de tierra.

\section{Espacialización de la información}

La información descrita caracteriza procesos de naturaleza continua o discreta. Por ejemplo, los datos continuos en el espacio son mediciones tomadas en puntos fijos pero de naturaleza continua, verbigracia la contaminación del aire y del suelo. Los procesos discretos en el espacio se refieren a la localización de eventos como los domicilios de los niños a quienes se les midieron los niveles de plomo en sangre. La espacialización de estos datos mediante modelos, facilita la identificación de patrones para conocer si la intensidad de los eventos varía sobre el área de estudio. No obstante, los mapas resultantes deben someterse sistemáticamente a pruebas estadísticas a fin de transformar la percepción visual en algo objetivo. De esta manera, a través del modelo espacial de riesgo se tiene la capacidad de representar y analizar la heterogeneidad de valores en sus dimensiones espaciales y temporales $(18,19)$.

Con el objeto de establecer patrones de mayor a menor nivel de plomo tanto en sangre como en el ambiente, se aplicó un modelo geo-espacial para estimar o predecir el valor del contaminante en un punto no muestreado, a partir de los valores conocidos de las muestras. A continuación se realizó un proceso de interpolación y de suavizamiento, para generar una superficie continua y llevar a cabo la superposi- ción temática. Los productos de este modelado fueron: 1) un mapa de isolíneas, donde cada una de éstas representa una concentración determinada del tóxico en el espacio, y 2) un mapa con información continua (figura 1).

\section{Áreas de influencia}

A fin de establecer zonas de influencia se consignaron anillos concéntricos alrededor de las posibles fuentes de contaminación. El producto generado fue un mapa de distancia. Se asumió una distancia máxima de $400 \mathrm{~m}$ para estratificar la localidad en dos zonas (dentro y mas allá de los $400 \mathrm{~m}$ ). Un procedimiento similar se realizó en el 
mapa de líneas que representa las calles pavimentadas, considerando un área de influencia de $100 \mathrm{~m}$. Esta información se utilizó en forma complementaria para identificar el área bajo influencia de calles de tierra.

\section{Superposición temática}

La superposición de información a través de mapas tiene la ventaja de analizar diferentes contenidos temáticos con el objeto de establecer patrones espaciales, ya sea en forma visual o analítica. En la figura 1 se enuncian los diferentes mapas generados, identificados según el nivel de análisis de la información que representan. Así por ejemplo, los mapas iniciales están compuestos por dos elementos fundamentales: una base geográfica o mapa base, común a todos los mapas (amanzanado de la ciudad), y una capa de contenido temático propio de cada uno, integrado al mapa mediante una planilla. A partir del análisis de los datos iniciales se obtuvieron los mapas derivados con los cuales, al haberles asignado algún criterio de clasificación, se generaron los mapas interpretados. Los mapas resultantes se crearon a partir de las combinaciones construidas con la información precedente, sobre la cual se tomarán diferentes decisiones.

\section{Análisis de asociación y estadística descriptiva}

Entre los diferentes análisis se realizó un procedimiento de asociación "exposición-efecto" a través del SIG-Epi, que brinda un conjunto de medidas y técnicas estadísticas para detectar la posible asociación entre un factor de exposición espacial y un efecto bajo estudio. Se definieron como factores de exposición tanto las áreas de influencia generadas por la proximidad a un "sitio-fuente" como las zonas con calles de tierra. Ambos fueron representados a través de mapas. Con respecto al mapa de efecto, se utilizaron los domicilios de los niños con plombemias iguales o superiores a
$10 \mu \mathrm{g} / \mathrm{dL}$, consideradas como efecto no deseado $(20,9)$.

Para la asociación entre los datos de plomo ambiental y de las plombemias, se generó un área de influencia de $100 \mathrm{~m}$ alrededor de las muestras de polvo ambiental, con el objeto de capturar los datos provenientes de una iso-línea de plombemias que cruzara por dicha área. Esta información se relacionó mediante un diagrama de cajas (box-plot), con el fin de resumir medidas de tendencia central y dispersión. El rectángulo del diagrama de cajas representa el intervalo entre cuartiles, y la línea dentro del mismo la mediana. Las líneas verticales representan 1,5 veces el valor del rango intercuartil por encima y por debajo de los respectivos cuartiles.

\section{RESULTADOS}

Con respecto a los factores ambientales en la ciudad de SAO, a través de la escena satelital se observa la disposición a cielo abierto de los residuos de la antigua fundición. Mediante inter- pretación visual y verificación posterior en terreno, se constata su presencia y dispersión a través de la erosión eólica e hídrica. Sumado a esto, se aprecia la existencia de cavas. La vecindad entre los residuos de la fundición y la extracción de áridos para la construcción, hace suponer que existe mezcla de materiales (figura 2).

Durante el periodo activo de la fundición (1965-1985), además de la acumulación de residuos se generó particulado proveniente de los hornos de fundición de la ciudad. Este escenario pudo dar lugar a otra vía de exposición, ya que a menos de $100 \mathrm{~m}$ se localiza el tanque de agua de la ciudad. Si bien el agua de uso residencial es externa al sitio, en una etapa del proceso de distribución pasa por piletas a cielo abierto. En la actualidad el líquido podría estar contaminado con el particulado trasladado a través del viento.

La exposición al plomo ambiental puede ocurrir por diversas vías; aquí sólo se consideraron aquellos sectores donde el particulado en suspensión puede generar mayor probabilidad de exposición aérea. La localidad fue zo-

FIGURA 2. Localización de posibles fuentes de contaminación, sobre imagen Aster. San Antonio Oeste, Río Negro, Argentina, 2006
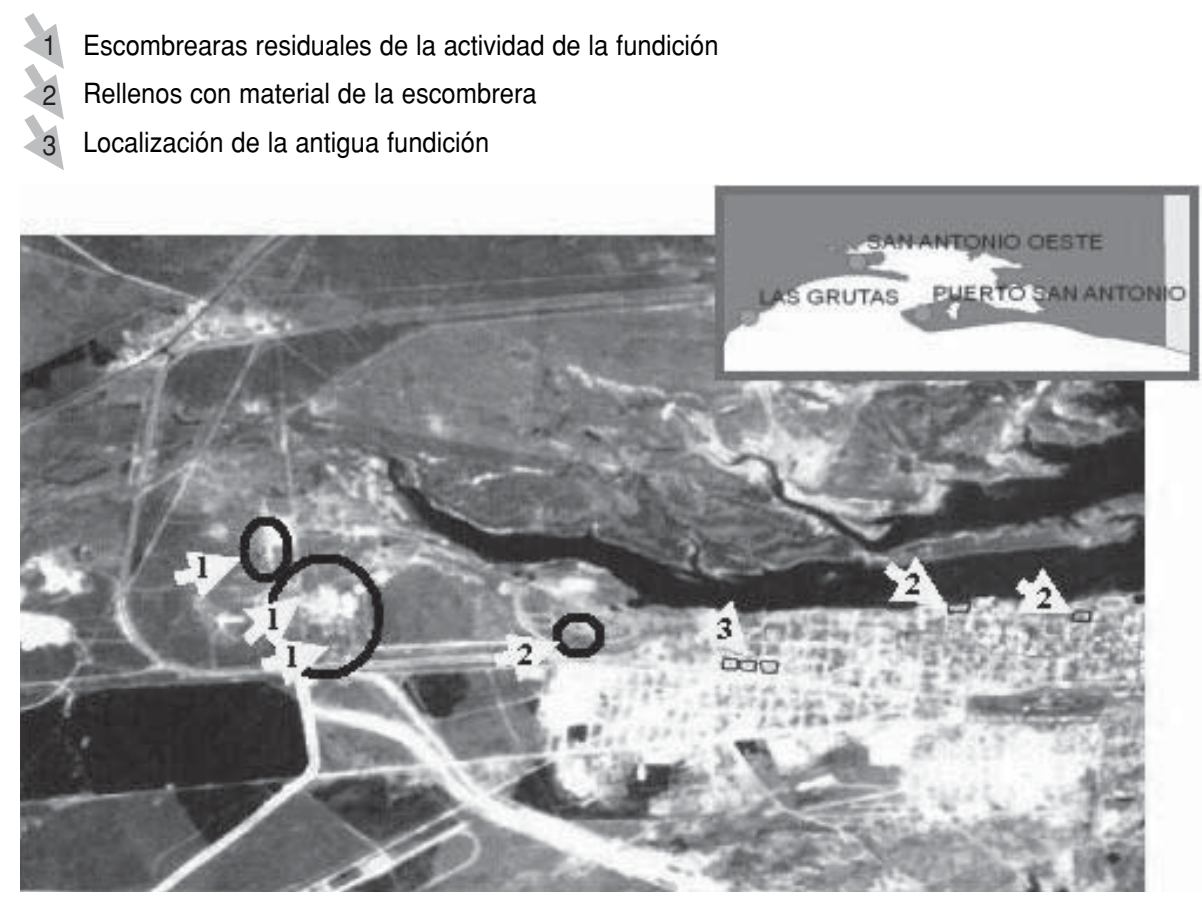
FIGURA 3. Ubicación domiciliaria de 186 niños con medición de niveles de plomo en sangre. San Antonio Oeste, Río Negro, Argentina, 2006

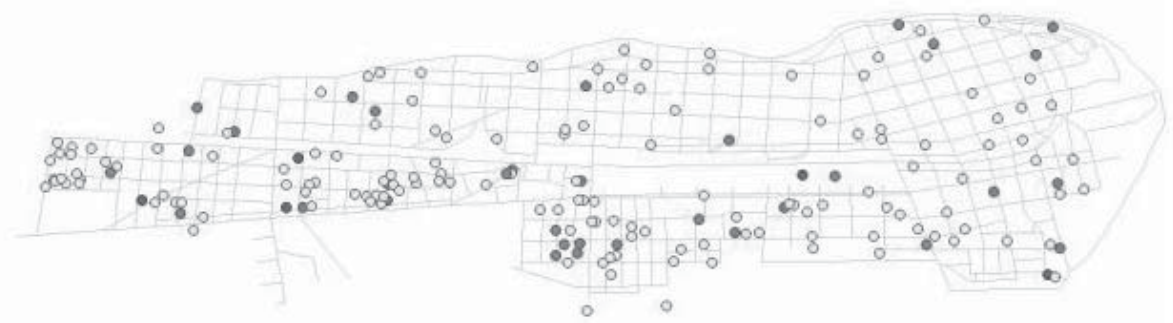

Plombemias según tipo de intervención
○ $0001-9999$
$10000-13999$
- 14 000-50 999

FIGURA 4A. Espacialización de datos de plomo en sangre. Desarrollo de superficie continua por interpolación de iso líneas. San Antonio Oeste, Río Negro, Argentina, 2006

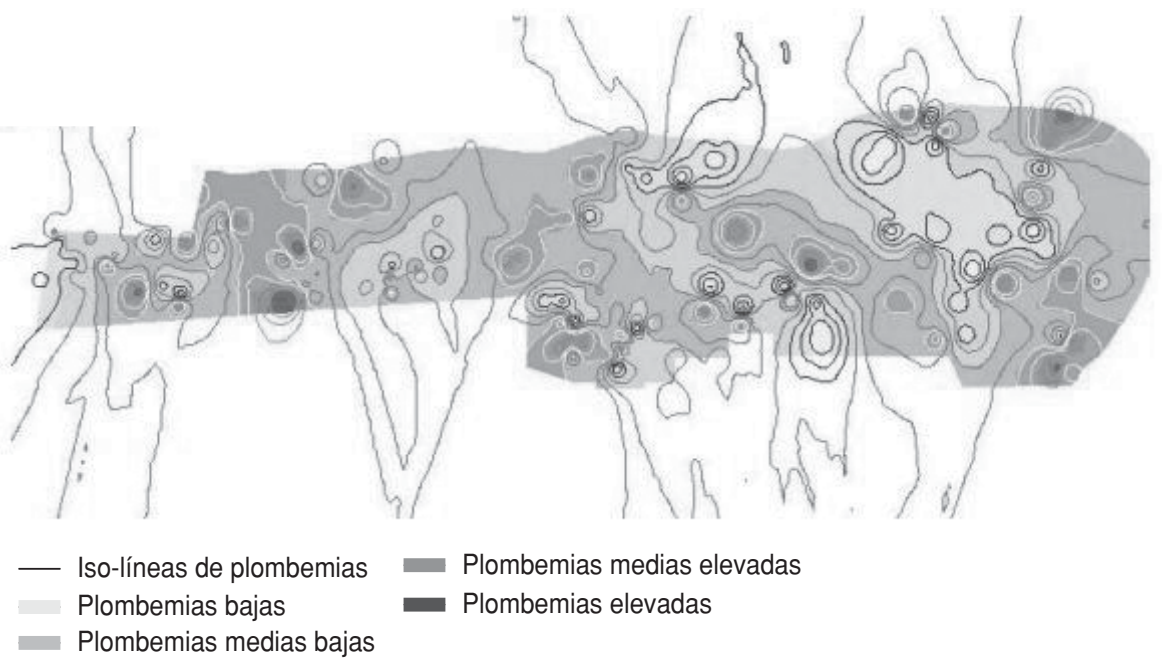

FIGURA 4B. Áreas delimitadas según estimación de los valores de plombemia $\geq 10 \mu \mathrm{g} / \mathrm{dL}$. San Antonio Oeste, Río Negro, Argentina, 2006

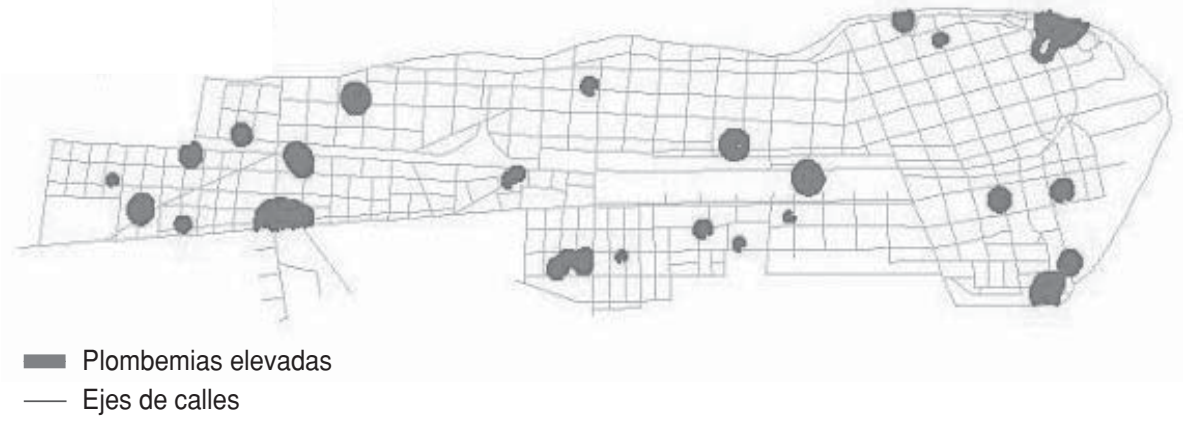

nificada según la proximidad de las viviendas o escuelas a un sitio potencial de dispersión y/o su localización sobre calles no pavimentadas.

La distribución de los domicilios de los niños a quienes se extrajo sangre no muestra una disposición espacial de sencilla interpretación (figura 3), razón por la cual se generaron núcleos de plombemias no deseadas (figuras 4a y 4b) para su confrontación con los factores de exposición antes mencionados. Esto es posible a partir de la generación de iso-líneas que representan niveles similares de plomo en sangre y la extracción únicamente de aquellos puntos donde los valores son superiores a $10 \mu \mathrm{g} / \mathrm{dL}$. Los 23 núcleos definidos equivalen a 38 domicilios de residencias detectados con, al menos, una plombemia elevada. La exposición al plomo puede darse tanto en el domicilio residencial como en el escolar, debido a la larga permanencia de los niños en la escuela (figura 5).

En cuanto a la proximidad a los sitios-fuente, de los 38 domicilios de residencias con al menos una plombemia elevada, se identificaron 10 niños con plombemias no deseadas con residencia dentro de áreas de influencia. Asimismo, tres de las seis escuelas se ubican en algún área de influencia, y se observó más abundancia de plombemias mayores o iguales a $10 \mu \mathrm{g} / \mathrm{dL}$ $(n=22)$ en estas escuelas con respecto al resto (figura 6). En seis casos los niños tendrían las dos posibles exposiciones. Con respecto al tipo de calle, aquellas que son de tierra agrupan 31 casos con plombemias no deseadas, frente a siete de las pavimentadas (figura 7). La distribución de los núcleos con plombemias elevadas en relación a la proximidad a las calles de tierra y/o sitios fuente se muestra en la figura 8 . El análisis de la asociación-efecto fue significativa (RP=6,48; IC95\%: 1,29-32,36). Es decir que residir o permanecer durante un tiempo prolongado (como en el caso de la asistencia al colegio) sobre una calle de tierra y/o en la vecindad de un sitio-fuente, genera un riesgo estadísticamente significativo. En cuanto a los valores de plomo ambiental que se determinaron en las muestras obtenidas, se identificó una zona de riesgo coinci- 
FIGURA 5. Distribución de escuelas y domicilios de niños con plombemia elevada. San Antonio Oeste, Río Negro, Argentina, 2006

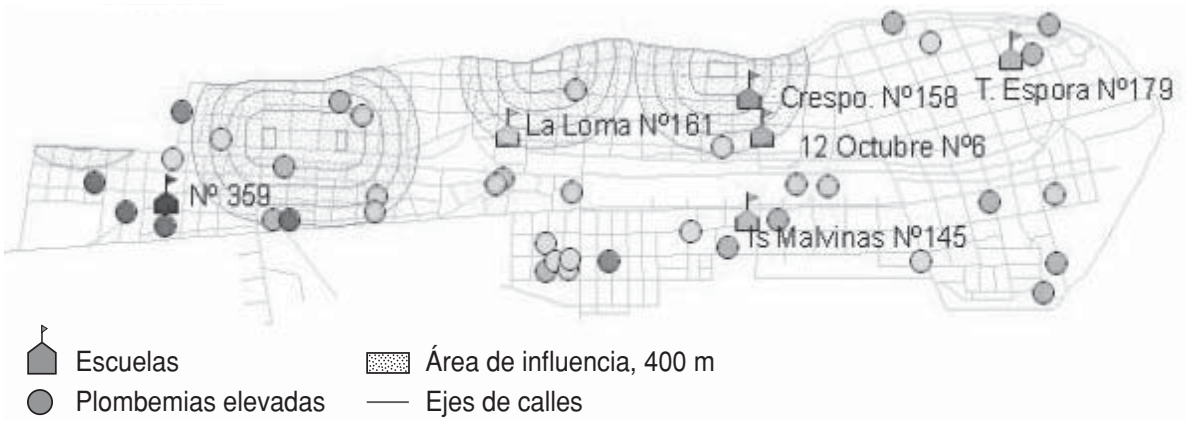

FIGURA 6. Distribución de plombemias agrupadas por escuela. San Antonio Oeste, Río Negro, Argentina, 2006

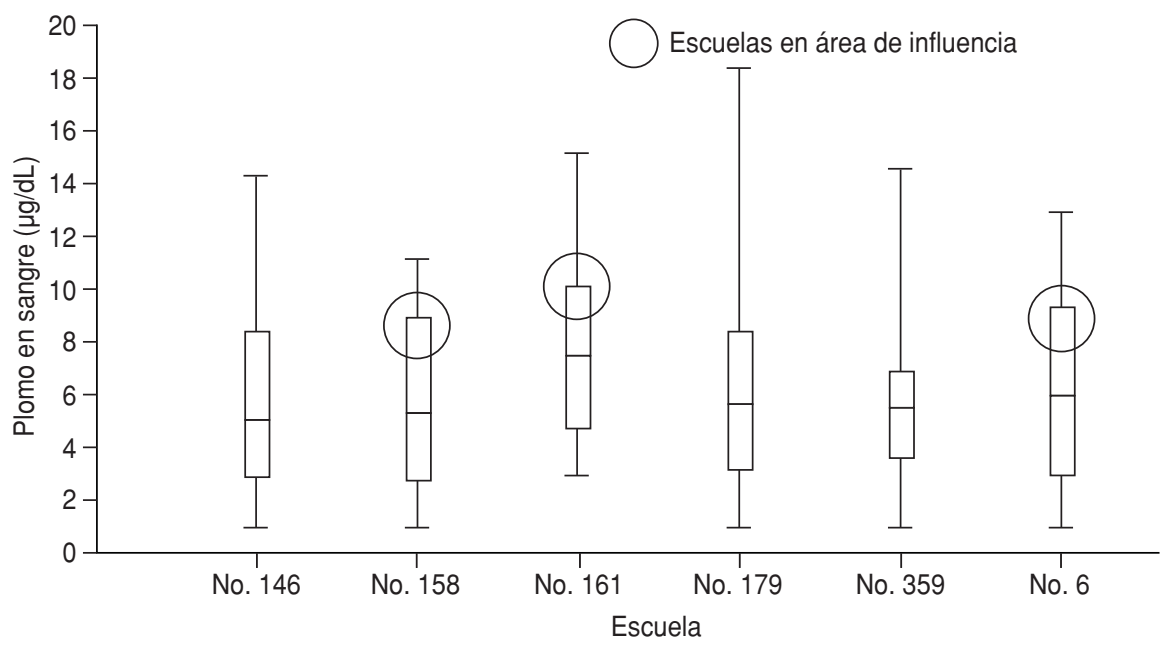

FIGURA 7. Frecuencia de plombemias elevadas por tipo de calle. San Antonio Oeste, Río Negro, Argentina, 2006
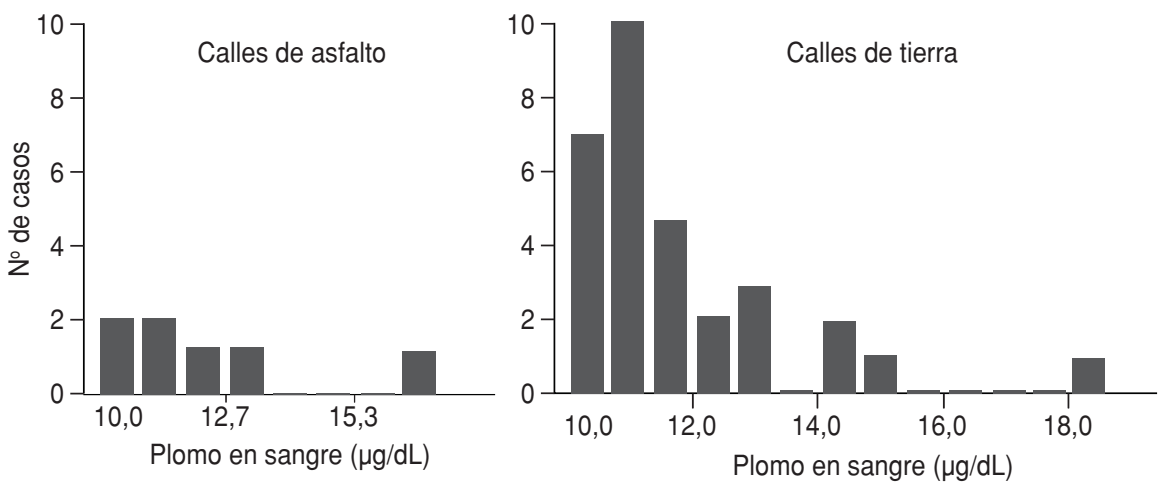

dente con la ubicación de la antigua fundición (figura 9).

A pesar de no existir correspondencia entre el número, localización y mo- mento del relevamiento de polvo con la extracción de muestras de sangre de los niños, fue posible analizar su vinculación a través de las estimaciones extraí- das de los modelos geo-espaciales. Los valores más elevados de plomo en sangre corresponden a rangos más altos de plomo ambiental. En la figura 10 se muestra un diagrama de cajas por grupos de plombemias. Los umbrales de corte de plomo en sangre fueron hasta de $4 \mu \mathrm{g} / \mathrm{dL}, 4-6 \mu \mathrm{g} / \mathrm{dL}$ y $\geq 7 \mu \mathrm{g} / \mathrm{dL}$, definidos por el método de corte natural (Jenks). Los valores de la mediana de polvo ambiental fueron 68, 83,9 y $150,2 \mu \mathrm{g} / \mathrm{g}$ pero sólo en el último grupo se consignaron valores por encima de 400 ppm (límite establecido por EPA para suelo en áreas de juego infantil descubiertas) (21).

\section{DISCUSIÓN}

El análisis desarrollado en este trabajo mostró que las plombemias elevadas estuvieron asociadas con la proximidad a algún sitio-fuente y/o localización de los domicilios residenciales o escolares sobre calles de tierra. Las muestras de sangre infantil tomadas en las escuelas ubicadas en el área de influencia de alguna fuente, presentaron mayor abundancia de plombemias elevadas que las localizadas en otra área. Por último, los valores de plomo en sangre más elevados correspondieron a rangos de plomo ambiental más altos.

Si bien el método óptimo para medir los niveles del contaminante en el receptor es la cuantificación en el medio ambiental involucrado, asociado con la ruta potencial de exposición durante el periodo crítico de exposición, pocas veces se presenta la oportunidad de realizar tal medición. Por esta razón, en forma alternativa se predijo el nivel ambiental mediante el modelado de la fuente-receptor.

La dificultad metodológica de este tipo de estudio radica en poder captar la variabilidad temporal de situaciones como: a) la movilidad de los niños potencialmente expuestos por mudanzas o por la propia rutina diaria entre el hogar, la escuela y los espacios de recreación, entre otros, lo que lleva a un gran número de situaciones difíciles de medir, y b) las tendencias y los cambios de incidencia o duración de exposición 
FIGURA 8. Distribución de núcleos con plombemias elevadas por tipo de calles y área de influencia de las fuentes. San Antonio Oeste, Río Negro, Argentina, 2006

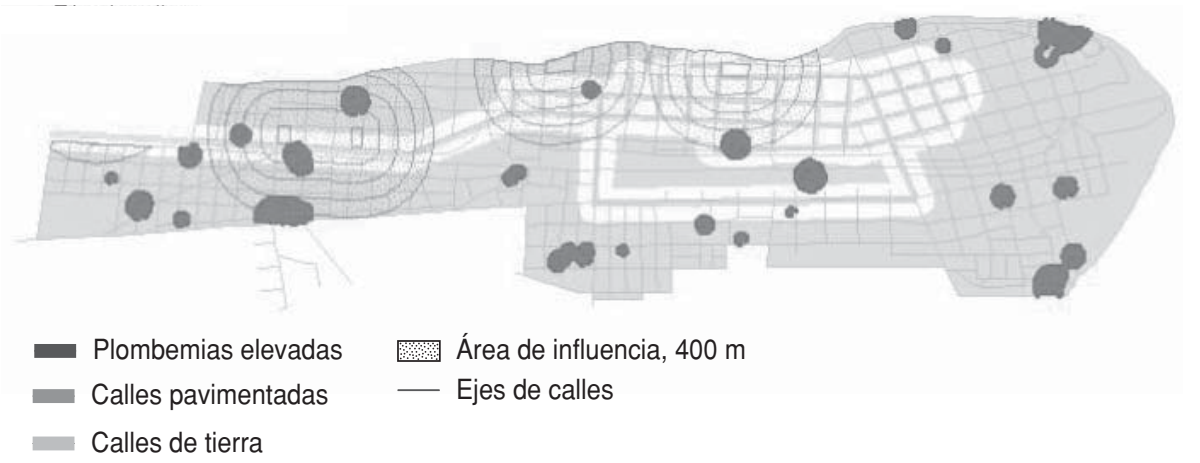

FIGURA 9. Distribución de las muestras de polvo ambiental e iso-líneas generadas según la concentración de plomo ambiental. San Antonio Oeste, Río Negro, Argentina, 2006

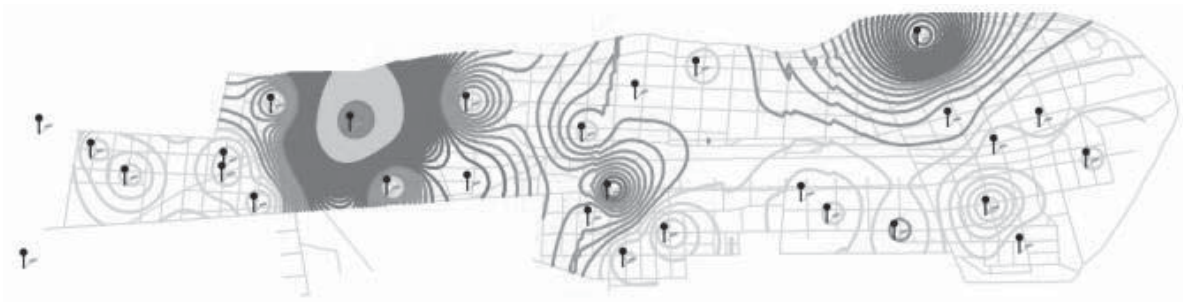

Plomo en polvo ambiental

10-70 - 401-800

-71-140-801-1030

- 141-400 is Muestras

FIGURA 10. Gráfico de cajas de los valores de plomo ambiental por grupos de plombemias definidos por el método de corte natural (Jenks). San Antonio Oeste, Río Negro, Argentina, 2006

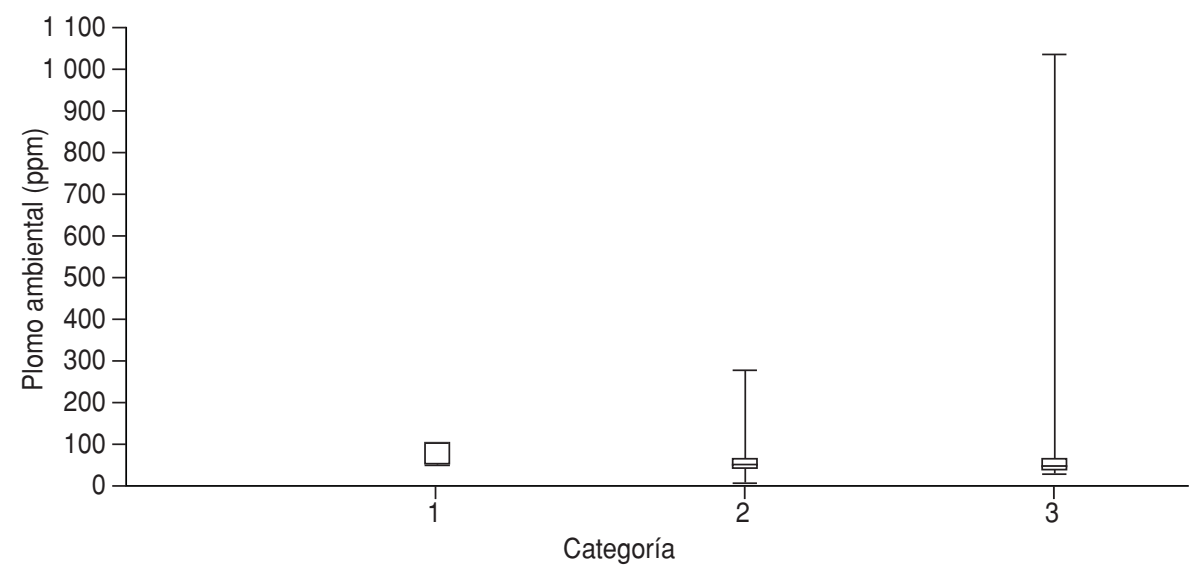

que imponen las estaciones del año, las condiciones meteorológicas y los ciclos de producción y, por consiguiente, de generación de residuos. minante entre la fuente y el receptor es factible hallar la asociación entre la fuente de contaminación y los biomarcadores de exposición en los individuos. Se ha descrito de manera vasta $(23,24)$ el hallazgo de mayores niveles de plomo en sangre de niños que habitan las viviendas más próximas a una fundición, como también la correlación con el contenido de plomo en el polvo intradomiciliario y el polvo ambiental de patios, huertas y jardines. Consideraciones similares se han realizado en la evaluación del efecto de, por lo menos, 2000 demoliciones de edificios durante el periodo 2001-2002 en las plombemias de los niños de St. Louis, EUA (25). Con respecto al estudio de $\mathrm{SAO}$, los valores de plombemias no deseadas mostraron una prevalencia igual a $20 \%$.

Un estudio realizado con 203 niños de 3 meses a 13 años de edad, en la ciudad de Buenos Aires, en 2000, mostró una prevalencia igual a 9,6\% (26). Otra investigación que se llevó a cabo en la ciudad argentina de Córdoba, en 1996, con 172 niños de 6 meses a 9 años de edad, indicó una prevalencia de $26.7 \%$ (27). Al comparar estos estudios cabe tomar en cuenta que la eliminación del plomo de las gasolinas en Argentina data de 1996, y que según las referencias internacionales ésa era una de las principales fuentes de exposición al metal (28).

Asimismo, en varios países de América Latina se han usado, hasta hace pocos años, las gasolinas con plomo con un alto potencial contaminante y las restricciones al uso y disposición final de plomo datan de fechas recientes. En este escenario es normal encontrar en la población infantil una distribución de plombemias superiores a $10 \mu \mathrm{g} / \mathrm{dL}$ que oscila entre $5 \%$ y $30 \%$ de los niños estudiados (13).

Investigaciones llevadas a cabo en otras naciones muestran una gran variabilidad: en México 76\% de los niños tuvieron concentraciones de plomo en sangre mayores a $10 \mu \mathrm{g} / \mathrm{dL}$, y los principales predictores fueron el uso de cazuelas y ollas de barro vidriado para cocinar y guardar los alimentos. En 1999, la prevalencia de plombemias superiores a $10 \mu \mathrm{g} / \mathrm{dL}$ en los niños de 
12 a 14 años que vivían en la Región Metropolitana de Santiago de Chile fue de $1,2 \%$ y para los niños de 4 a 5 años de edad de 1,4\%. En Montevideo, en 1994 fue de 41,7\%, y en 2004 descendió a $6,7 \%$ para individuos menores de 18 años de edad, luego de que se eliminaran los aditivos con plomo de las gasolinas (29).

Este trabajo resalta la importancia de definir otros criterios relacionados con el análisis espacial para el diagnóstico de un sitio contaminado. Además del valor de la medición u observación, los datos espaciales incluyen la localización o posición relativa de los valores observados en el entorno, lo cual no se obtiene a través de encuestas ni surge del análisis univariado de las variables tal como son estudiadas por los métodos epidemiológicos clásicos. En un estudio descriptivo (30) sin considerar la espacialidad de la información, es extremadamente difícil obtener significación estadística debido a la complejidad de las variables involucradas, y a la dificultad para cuantifi- carlas y representarlas como entidades espacio-temporales.

El análisis espacial generado a partir de la georreferenciación de los domicilios de casos y fuentes, añadió una nueva dimensión al estudio descriptivo al brindar más flexibilidad para el manejo de los datos. Facilitó el establecimiento del contexto en el cual el evento de salud ocurre, contribuyendo a la comprensión de procesos socio ambientales al trasladar datos e información entre unidades espaciales. El SIG facilitó la visualización simultánea de diferentes capas de información sobre factores relacionados con los problemas de salud estudiado, asociados a un territorio específico, haciendo evidentes, por simple interpretación visual, las relaciones y los fenómenos. A través del análisis espacial se encontró la asociación entre plombemias elevadas y la proximidad a la fuente y el hecho de vivir en calles de tierra. Los resultados aportaron la evidencia científica necesaria para indicar la necesidad de llevar a cabo medidas de inter- vención directas sobre las fuentes de exposición y de educación para fomentar hábitos dietéticos higiénicos en la población.

Agradecimientos. Los autores agradecen la información cedida para la elaboración de este trabajo a Elena Pedroni, de la Dirección de Epidemiología del Ministerio de Salud de la Nación, Argentina; José Luis Estévez, del Centro Nacional Patagónico del CONICET, Argentina; Fernando Díaz Barriga, del Departamento de Toxicología Ambiental de la Facultad de Medicina de la Universidad Autónoma de San Luis Potosí, México, y Bonuccelli de la Universidad Nacional de La Pampa, Argentina. Asimismo, agradecen la colaboración de Mirta Carvajal, de la Fundación Inalafquen, y Maite Narvarte, del Instituto de Biología Marina y Pesquera "Almirante Storni", así como de la Fundación Patagonia Natural, en Río Negro, Argentina.

\section{REFERENCIAS}

1. Goldenberg JS, Contreras Escudero L. Diseño y puesta en marcha de un sistema de vigilancia epidemiológica en salud mental. Rev Panam Salud Publica. 2002;11(2):83-92.

2. Hills MA. Statistical methods used in assessing the risk of disease near a source of possible environmental pollution: a review. J R Stat Soc Asssoc. 1989;152:353-63.

3. Bastos Fl, Barcellos CA. Geografía social de AIDS no Brasil. Rev Saúde Publ. 1995;29(1): $52-62$.

4. Barcellos C. Unidades y escalas en los análisis espaciales en salud. Rev Cubana Salud Pública. 2003;29(4):307-13.

5. Bonuccelli R, Malán JM, Luna L, Torres 1 . Contaminación por metales pesados derivados de la lixiviación de escorias de fundición. San Antonio Oeste, Río Negro. Actas del Congreso Geológico Argentino de La Plata. Tomo IV. 2005.

6. Gil M, Commendatore M, Harvey M, Colombo JC, Estévez JL. Evaluación de la contaminación por hidrocarburos y metales en la zona costera patagónica. Plan de Manejo Integrado de la Zona Costera Patagónica. Informe Técnico 21. Puerto Madryn, Argentina; 1996.

7. Departamento Provincial de Aguas. Dirección de Protección y Conservación de los Recursos Hídricos-DGRH. "Evaluación de la contaminación por metales pesados en la Bahía de San Argentina; 2000.
Antonio". Informe final. Viedma, Río Negro,

8. Gil M, Harvey M, Beldoménico H, García S, Commendatore M, Gandini P, et al. Contaminación por metales y plaguicidas organoclorados en organismos marinos de la zona costera patagónica. Plan de Manejo Integrado de la Zona Costera Patagónica. Informe Técnico 32. Puerto Madryn, Argentina; 1997.

9. US Centers for Disease Control and Prevention. Department of Health and Human Services. Screening young children for lead poisoning. Guidance for state and local public health officials. Atlanta, GA;1997.

10. US Environmental Protection Agency. Testing your home for lead. In paint, dust and soil. EPA 747-k-00-001. July 2000.

11. US Environmental Protection Agency. Residential Lead Hazard Standards-TSCA Section 403. Federal Register, January 2001.

12. NTOXMET Workshop. The declaration of Brescia on prevention of the neurotoxicity of metals. Brescia, Italia 17-18 June 2006. [Sitio en Internet] Hallado en: http://www. ntoxmet.it. Acceso el 19 noviembre 2006.

13. Yañez L, Ortiz D, Calderón J, Batres L, Carrizales L, Mejia J, et al. Overview of human health and chemical mixtures: problems facing developing countries. Environ Health Perspect. 2002;110 (supl 6):901-9.
14. Instituto Nacional de Estadísticas y Censo. Localidades por fracción, radios y ejes de calles con nombres. Datos de población y vivienda de San Antonio Oeste. Río Negro, Argentina; 2001.

15. Organización Panamericana de la Salud. Sistema de información geográfica en epidemiología y salud pública. Versión 1.26. Washington, DC: OPS; 2003.

16. Organización Panamericana de la Salud. Uso de sistemas de información geográfica en epidemiología (SIG-Epi). Boletín Epidemiológico de la OPS. 1996;17(1). Hallado en: URL: http://www.paho.org/spanish/sha/BE_ v17n1.pdf. Acceso el 3 enero 2005.

17. Programa Nacional de Prevención y Control de Intoxicaciones del Ministerio de Salud y Ambiente de la Nación. Intoxicaciones con plomo en niños. Guía de diagnóstico y tratamiento. Buenos Aires; febrero 2006.

18. Maskrey A. Navegando entre brumas. La aplicación de los sistemas de información geográfica al análisis de riesgo en América Latina. Red de Estudios Sociales en Prevención de Desastres en América Latina. Ciudad de Panamá; 1998.

19. López A, Ortega G. Aplicación de técnicas de análisis espacial a la mortalidad por cáncer en Madrid. Ârea de Epidemiología Ambiental y Cáncer. Centro Nacional de Epidemiología. 
Documentos Técnicos de Salud Pública No. 66. Madrid, España. Hallado en: http:// www.scielo.isciii.es/mono/pdf/CNE_03.pdf. Acceso el 17 abril 2006.

20. Garcia S. Exposición crónica a plomo y conducta violenta. Buenos Aires, Argentina; 2006. Hallado en: http://www.msal.gov.ar/ redartox/mateducativo/pb.ppt. Acceso el 18 noviembre 2006.

21. US Environmental Protection Agency. Residential lead hazard standards. TSCA Section. Washington, DC; 2005. Hallado en: http:// www.epa.gov/lead/pubs/leadhaz.htm. Acceso el 20 septiembre 2006.

22. Nuckols JR, Ward MH, Jarup L. Using geographic information systems for exposure assessment in environmental epidemiology studies. Environ Health Perspect. 2004;112(9): 1007-15.

23. Ordóñez BR, Romero LR, Mora R. Investigación epidemiológica sobre niveles de plomo en la población infantil y en el medio ambiente domiciliario de Ciudad Juárez, Chihuahua, en relación con una fundición de El Paso, Texas. Salud Publica Mex. 2003;45(supl 2): S281-95.

24. Willmore A, Sladden T, Bates L, Craig BD. Use of a geographic information system to track smelter-related lead exposures in children: North Lake Macquarie, Australia 1991-2002. International Journal of Health Geographics. 2006, 5:30. Hallado en: http:// www.ij-healthgeographics.com/content/ 5/1/30. Acceso el 6 diciembre 2006.

25. Patridge J, Schneider RH, Gibson R, Phillips $\mathrm{P}$, Rabito F, Shorter $\mathrm{Ch}$, et al. Using spatial analyst to determine effects of demolition on blood lead levels in St. Louis City children. A preliminary look. Missouri Department of Health and Senior Services. Hallado en: http://www.dhss.mo.gov/EPHT/MOarticle. pdf. Acceso el 17 noviembre 2006.

26. Rovirosa A, Uicich R, Fernández Álvarez A, Sánchez de la Puente M, O'Donnell A. Niveles de plomo en sangre de niños de Buenos Aires. Congreso de Pediatría. Salta, Argentina; 2000.
27. Hansen C, Buteler R, Procopovich E, Pagan G, Díaz B, Gait N. Niveles de plomo en sangre de niños de la Ciudad de Córdoba. Medicina (Buenos Aires). 1999;59:167-70.

28. García S, Mercer R. Salud infantil y plomo en Argentina. Salud Publica Mex. 2003; 45 (supl 2):S252-55.

29. Mañay N. Contaminación por plomo en Uruguay. Seminario Taller sobre Sitios Contaminados con Plomo y Salud Humana. Santa Fe, Argentina, 23 de noviembre 2006.

30. Programa de Especialización en Epidemiología de Campo. Investigación de probable contaminación por plomo en escolares de San Antonio Oeste. Ministerio de Salud de Nación. Informe Técnico. Enero 2006.

Manuscrito recibido el 26 de diciembre de 2006. Aceptado para publicación, tras revisión, el 8 de diciembre de 2007.

ABSTRACT Objectives. To produce a geospatial model to evaluate lead exposure among school children from 6-8 years of age, in San Antonio Oeste, Rio Negro province, Argentina, an area contaminated by a foundry in the city center whose toxins were released into

\section{Geospatial models for local health surveillance}

the open air.

Methods. The spatial analysis conducted from October-April 2006 included satellite interpretation and mapping of the data to geographically plot the information. Residences on dirt roads were included, as was the distance for each of the study children's homes and/or schools to the site identified as the source of the exposure.

Results. Blood samples taken from children attending schools within the area surrounding the source showed higher lead levels than that of children in other areas. These lead levels were associated with the proximity to the source and/or with living on a dirt road. The highest blood lead levels corresponded to the higher environmental lead levels.

Conclusions. Spatial analysis was shown to be a useful tool for site analysis and risk management since it indicated a definitive association between elevated lead levels and the proximity to the source, and/or residing on a dirt road, connections which had not been revealed with traditional epidemiological analyses. The results provided the scientific evidence needed to begin implementing interventions regarding the sources of exposure and education aimed at promoting more hygienic dietary habits among the population.

Key words Geographic Information Systems, environmental exposure, blood, lead, child welfare, Argentina. 